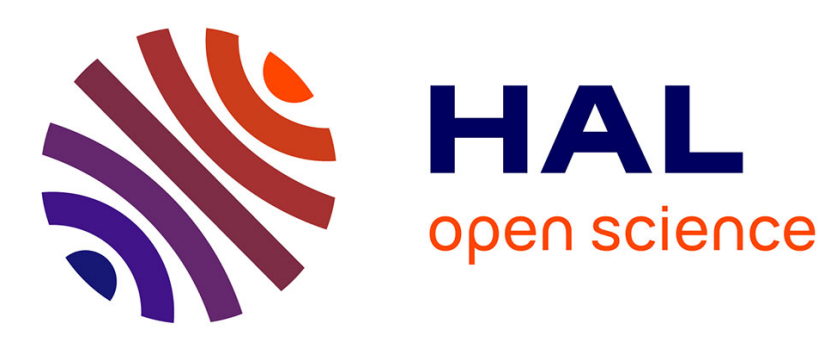

\title{
Defaulting firms and systemic risks in financial networks
}

Nicolas Houy, Frédéric Jouneau

\section{To cite this version:}

Nicolas Houy, Frédéric Jouneau. Defaulting firms and systemic risks in financial networks. 2016. halshs-01267340

\section{HAL Id: halshs-01267340 \\ https://shs.hal.science/halshs-01267340}

Preprint submitted on 4 Feb 2016

HAL is a multi-disciplinary open access archive for the deposit and dissemination of scientific research documents, whether they are published or not. The documents may come from teaching and research institutions in France or abroad, or from public or private research centers.
L'archive ouverte pluridisciplinaire HAL, est destinée au dépôt et à la diffusion de documents scientifiques de niveau recherche, publiés ou non, émanant des établissements d'enseignement et de recherche français ou étrangers, des laboratoires publics ou privés. 
UMR 5824

\author{
93. chemin des Mouilles \\ 69130 Ecully - France \\ Maison de IUniversité, Bâtiment $\mathrm{B}$ \\ 10, rue Trefilerie \\ 42023 Saint-Etienne cedex $02 \cdot$ France \\ http://www.gate.cnis.fr \\ gate gate.cnrs.fr \\ WP 1606 - February 2016
}

\title{
Defaulting firms and systemic risks in financial networks
}

\author{
Nicolas Houy, Frédéric Jouneau
}

\begin{abstract}
:
In this paper, we use the axioms introduced in Eisenberg and Noe (2001) and Rogers and Veraart (2013) and study their consequences in terms of optimal sets of defaulting firms. We show that, from this point of view, the Absolute Priority axiom is not independent. We also show that the optimal sets of defaulting firms characterized in Eisenberg and Noe (2001) are still optimal when the Limited Payment axiom, implicit in Eisenberg and Noe (2001), is further removed. However, some other optimal sets of defaulting firms appear in this case. Finally, with the help of counterexamples, we show that no further weakening in the set of axioms considered can lead to positive results.
\end{abstract}

\section{Keywords:}

Credit Risks, Systemic Risks, Clearing Systems, Financial System

JEL codes:

G21, G32, G33

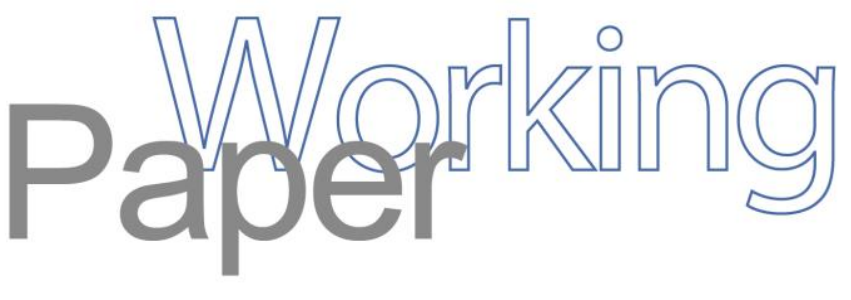




\title{
Defaulting firms and systemic risks in financial networks
}

\author{
Nicolas HouY* Frédéric JounEAU ${ }^{\dagger}$
}

February 4, 2016

\begin{abstract}
In this paper, we use the axioms introduced in Eisenberg and Noe (2001) and Rogers and Veraart (2013) and study their consequences in terms of optimal sets of defaulting firms. We show that, from this point of view, the Absolute Priority axiom is not independent. We also show that the optimal sets of defaulting firms characterized in Eisenberg and Noe (2001) are still optimal when the Limited Payment axiom, implicit in Eisenberg and Noe (2001), is further removed. However, some other optimal sets of defaulting firms appear in this case. Finally, with the help of counterexamples, we show that no further weakening in the set of axioms considered can lead to positive results.
\end{abstract}

Key-Words: Credit Risks, Systemic Risks, Clearing Systems, Financial System. JEL Classification: G21, G32, G33.

\footnotetext{
${ }^{*}$ University of Lyon, Lyon, F-69007, France; CNRS, GATE Lyon Saint-Etienne, F-69130, France. Email: houy@gate.cnrs.fr.

${ }^{\dagger}$ Université de Lyon, Lyon, F-69007, France ; CNRS, GATE Lyon Saint-Etienne, Ecully, F-69130, France; Université Lyon 2, Lyon, F-69007, France. E-mail: jouneau@gate.cnrs.fr.
} 


\section{Introduction}

Over the last ten years, increasing attention has been paid to the interconnections in financial institutions networks and at their consequences on credit risks and failures. Though not always precisely defined, systemic risk has become a "buzz word" for many studies in numbers of fields ranging from micro-structure of financial markets to monetary policies and regulation rules. Much efforts have been devoted to measure the consequences of contagious failures, assessing their likelihood in actual financial networks, evaluating the efficiency of mitigating policies such as the control of capital flows (according to the Basel agreements), the comprehensive use of central clearing houses (according either to the European EMIR or the US Dodd-Frank act regulation rules) or the bailout commitment by lenders of last resort. Allen and Gale (2000) first addressed the question by specific examples (see also Acemoglu et al. (2015)). They showed in particular that a trade-off may arise between the lowering of risks at an individual level allowed by diversification and the implied increased risk of contagion at the industry level (this line of work has been further investigated by Battiston et al. (2012a,b)). Several attempts have been made to enlarge the initial scope either by looking at real-life examples (see, among others, Elsinger et al. (2006); Gauthier et al. (2012)) or by simulations (see, for instance Nier et al. (2007); Alessandri et al. (2009); Georg (2013)). ${ }^{1}$

However, as usual for studies with network structures, it is particularly difficult to provide clear-cut general results that apply whatever the graph considered. In this respect, the work by Eisenberg and Noe (2001) has emerged as a building stone for many papers. This work deals with a pure resolution problem (it is explicitly an ex-post framework) in which financial institutions must proceed to monetary exchanges compatible with pre-committed agreements and initial available endowments in operating cash flow. The purpose of Eisenberg and Noe (2001) is to derive the existence of a Clearing Payment Matrix (CPM), whose

\footnotetext{
${ }^{1}$ For a critical assessment of simulations in this literature, see Upper (2011).
} 
positive entries are the gross payments made from one institution to another and satisfying some constraints. First, Eisenberg and Noe (2001) state those constraints claiming that "[their] model satisfies the standard conditions imposed by bankruptcy law". These constraints are embedded by means of 4 axioms. Three are explicitly stated - Limited Liability (LL), Absolute Priority (AP) and Proportionality (P) - and another one is implicit in the solution concept - we call it Limited Payments (LP). Some of these axioms have been widely discussed and challenged. AP has been relaxed to account for possible haircuts and sunk costs (Rogers and Veraart (2013)), LL has been refined to deal with the case of cross holdings (Helmut (2009)), and P must be modified for taking priorities among debt holders into accounts. ${ }^{2}$ Given the four above axioms, Eisenberg and Noe (2001) provide a proof of existence of a CPM and discuss the uniqueness of it. This original framework has been extended to account for possible negative operating cash flows by Shin (2009) and reinterpreted as a valuation model by Glasserman and Peyton Young (2015).

The main issue of the present paper is to examine the consequences of the axiomatic approach proposed by Eisenberg and Noe (2001) on the set of defaulting firms. The subprime mortgage crisis made clear that this set is of crucial importance. Indeed, recall that Merrill Lynch has been purchased, Goldman Sachs and Morgan Stanley (who previously bought Bear Stearn) benefited from emergency lines of credit by the Federal Reserve whereas Lehman Brother went bankrupt. A public bailout commitment raises of course difficult incentive issues, but the consequences of large bank runs were famously recalled by Ben Bernanke on September 18, 2008 with the dramatic statement: "if we don't do this, we may not have an economy on Monday". Hence, deciding which banks should be saved and which should not is by no mean an easy question. However, it is one that must be addressed. The direct gross financial consequences of possible bad decisions should not be

\footnotetext{
${ }^{2}$ In practice such priorities are the rule (for instance public institutions are served first) or may be part of a justice decision. To treat all debt holders on equal ground is merely a theoretical simplification.
} 
overlooked. Recall the Troubled Asset Relief Program (TARP) investment by the Federal Reserve accounts for more than 400 billions of US Dollars and lasted about 6 years. ${ }^{3}$

Of course, in Eisenberg and Noe (2001), the identity of the defaulting firms is a consequence of the characterized CPM. We make the choice in this paper to study axioms stated on the CPM in light of their consequences on the implied set of defaulting firms. The first question the present paper tackles is the independence of the axioms proposed by Eisenberg and Noe (2001) in terms of minimal (in the sense of inclusion) set of defaulting firms. More precisely, we ask whether removing one or several axioms allows to reach smaller (in the sense of inclusion) sets of defaulting firms. It turns out that AP is not independent of other axioms in this sense. This result holds true even if we consider a different AP and LL axioms as in Rogers and Veraart (2013). Second, we investigate the importance of the LP axiom implicitly imposed by Eisenberg and Noe (2001). Removing this axiom (while keeping LL and P) does not allow to find a smaller (in the sense of inclusion) set of defaulting firms than the one induced by the CPM characterized in Eisenberg and Noe (2001). However, we may find other sets of defaulting firms (possibly smaller in the cardinal - or some size-weighted - sense). We also discuss various other possible conjectures about the "usefulness" of other sets of axioms and provide several examples for the sake of completeness of our study.

The remainder of this paper is structured as follows. We display our model and explicit axioms in Section 2. We give results in Section 3. Section 4 concludes.

\section{Model}

Let $N=\{1, \ldots, N\}$ be a set of firms. $N$ is finite and fixed throughout this study. Notice that, for the sake of simplicity and with no risk of confusion, we write $N$ for both the set of

\footnotetext{
${ }^{3}$ This does not include the public takeover of Fannie Mae and Freddie Mac nor the so called "Maiden Lane Transactions" programs.
} 
firms and the cardinality of this set. Each firm $i \in N$ is initially endowed with an operating cash flow $e_{i}$. Let $\left(e_{i}\right)_{i \in N} \in \mathbb{R}_{+}^{N}$ be the operating cash flow vector, one for each firm. Let $\mathcal{E}=\mathbb{R}_{+}^{N}$ be the the set of all possible operating cash flow vectors. Firms are interconnected with a nominal liability matrix $\left(L_{i, j}\right)_{i, j \in N} \in \mathbb{R}_{+}^{N \times N}$ such that $\forall i \in N, L_{i, i}=0$. Then, for any two firms $i, j \in N, L_{i, j}$ is the nominal liability of firm $i$ towards firm $j$. Let $\mathcal{L}$ be the set of all possible nominal liability matrices. We introduce the following notation: $\forall S, T \in 2^{N}$, $L_{i, S}=\sum_{j \in S} L_{i, j}, L_{S, i}=\sum_{j \in S} L_{j, i}, L_{S, T}=\sum_{j \in T} L_{S, j}$ and $e_{S}=\sum_{j \in N} e_{j}$.

The first purpose of this study is the design of a clearing payment matrix (CPM), $X \in \mathcal{L}$. Let $e=\left(e_{j}\right)_{j \in N} \in \mathcal{E}$ and $L=\left(L_{i, j}\right)_{i, j \in N} \in \mathcal{L}$. Let $X \in \mathcal{L}$ be a CPM. We say that given $L$ and $X$ a firm is defaulting if its payments are not exactly equal to its nominal liabilities. We call $D(L, X)$ the set of defaulting firms. Formally,

$$
D(L, X)=\left\{i \in N, \exists j \in N, X_{i, j} \neq L_{i, j}\right\} .
$$

We call $N D(L, X)$ the set of firms that are not defaulting, i.e.,

$$
N D(L, X)=N \backslash D(L, X) .
$$

Finally, we say that a firm is level-0-defaulting if its value of the equity (total payments of others to it plus its operating cash flow minus its total payment to others) is negative when $L$ is used as a CPM. We call $\underline{D}(e, L)$ the set of level-0-defaulting firms. Formally,

$$
\underline{D}(e, L)=\left\{i \in N, L_{N, i}+e_{i}-L_{i, N}<0\right\} .
$$

Finally, we consider two real numbers $\alpha, \beta$ such that $0<\alpha, \beta \leq 1$ fixed throughout this study. $\alpha$ is interpreted as the fraction of the face value of the operating cash flow realized on liquidation. $\beta$ is interpreted as the fraction of the face value of the total nominal liability to it realized on liquidation.

In order to be acceptable, we impose that the CPM satisfy some axiomatic conditions. 
The following conditions have been introduced explicitly or implicitly in Eisenberg and Noe $(2001){ }^{4}$

\section{Axiom 1 (Limited Liability, LL)}

Let $e=\left(e_{j}\right)_{j \in N} \in \mathcal{E}$ and $L=\left(L_{i, j}\right)_{i, j \in N} \in \mathcal{L}$. Let $X=\left(X_{i, j}\right)_{i, j \in N} \in \mathcal{L}$ a CPM. X satisfies Limited Liability if

$$
\left\{\begin{array}{l}
\forall i \in N D(L, X), X_{i, N} \leq X_{N, i}+e_{i} \text { and }, \\
\forall i \in D(L, X), X_{i, N} \leq \beta \cdot X_{N, i}+\alpha \cdot e_{i}
\end{array} .\right.
$$

\section{Axiom 2 (Absolute Priority, AP)}

Let $e=\left(e_{j}\right)_{j \in N} \in \mathcal{E}$ and $L=\left(L_{i, j}\right)_{i, j \in N} \in \mathcal{L}$. Let $X=\left(X_{i, j}\right)_{i, j \in N} \in \mathcal{L}$ a CPM. X satisfies Absolute Priority if

$$
\forall i \in D(L, X), X_{i, N}=\beta . X_{N, i}+\alpha . e_{i} .
$$

\section{Axiom 3 (Proportionality, P)}

Let $e=\left(e_{j}\right)_{j \in N} \in \mathcal{E}$ and $L=\left(L_{i, j}\right)_{i, j \in N} \in \mathcal{L}$. Let $X=\left(X_{i, j}\right)_{i, j \in N} \in \mathcal{L}$ a CPM. X satisfies Proportionality if

$$
\forall i, j \in N,\left\{\begin{array}{l}
L_{i, N}>0 \Rightarrow X_{i, j}=\frac{L_{i, j}}{L_{i, N}} \cdot X_{i, N} \text { and, } \\
L_{i, N}=0 \Rightarrow X_{i, j}=0
\end{array} .\right.
$$

\section{Axiom 4 (Limited Payments, LP)}

Let $e=\left(e_{j}\right)_{j \in N} \in \mathcal{E}$ and $L=\left(L_{i, j}\right)_{i, j \in N} \in \mathcal{L}$. Let $X=\left(X_{i, j}\right)_{i, j \in N} \in \mathcal{L}$ a CPM. X satisfies Limited Payments if

$$
\forall i \in N, X_{i, N} \leq L_{i, N} .
$$

Limited Liability imposes that no firm should have a negative value of the equity, possibly even after liquidation in case of a default. Absolute Priority imposes that after payments are made, defaulting firms should be left with no value of the equity after liquidation. Proportionality imposes that the payments to other firms made by any firm

\footnotetext{
${ }^{4}$ For AP and LL, we use the version given in Rogers and Veraart (2013). The Eisenberg and Noe (2001)'s version of AP and LL correspond to the case $\alpha=\beta=1$.
} 
should be proportional to its nominal liabilities. Limited Payments imposes that the total payments made by a firm should not exceed its total nominal liability.

Let $e=\left(e_{j}\right)_{j \in N} \in \mathcal{E}$ and $L=\left(L_{i, j}\right)_{i, j \in N} \in \mathcal{L}$. Let $\Gamma$ be a set of axioms. Let $D \in 2^{N}$ be a subset of $N$. We say that $D$ is a $\Gamma$-solution to $(e, L)$ if there exists a $\mathrm{CPM} X=$ $\left(X_{i, j}\right)_{i, j \in N} \in \mathcal{L}$ such that

1. $X$ satisfies axioms in $\Gamma$,

2. $D=D(L, X) .^{5}$

We say that $D$ is an optimal $\Gamma$-solution to $(e, L)$ if $D$ is a $\Gamma$-solution to $(e, L)$ and for any $D^{\prime} \subset D, D^{\prime}$ is not a $\Gamma$-solution to $(e, L)$. In this study, we will focus on optimal solutions with one of the implicit objective of designing a CPM should be to try to minimize the set of defaulting firms.

\section{Results}

The following proposition sums up or generalizes some results already given in Eisenberg and Noe (2001) and Rogers and Veraart (2013) and that are relevant to our study in terms of optimal sets of defaulting firms.

\section{Proposition 1}

Let $0<\alpha, \beta \leq 1$. Let $e=\left(e_{j}\right)_{j \in N} \in \mathcal{E}$ and $L=\left(L_{i, j}\right)_{i, j \in N} \in \mathcal{L}$. The set of $\{L L, A P, P, L P\}$ solutions to $(e, L)$ is not empty. Moreover, if $D \in 2^{N}$ is an optimal $\{L L, A P, P, L P\}$ solution, then

1. $\underline{D}(e, L)=\emptyset$ if and only if $D=\emptyset$,

2. $\underline{D}(e, L) \subseteq D$.

\footnotetext{
${ }^{5}$ In this case, with a lack of rigor, but with no risk of confusion we may say that $D$ satisfies axioms in $\Gamma$ whereas it should be said that there exists a CPM $X$ satisfying axioms in $\Gamma$ such that that the set of defaulting firms, when $X$ is implemented, is $D$.
} 
Proposition 1-1 states that, if the nominal liability matrix $L$ can be implemented as a CPM without any defaulting firm, then $L$ trivially satisfies axioms LL, AP, P and LP and hence, $D=\emptyset$ is an optimal $\{\mathrm{LL}, \mathrm{AP}, \mathrm{P}, \mathrm{LP}\}$-solution. Obviously, the converse implication trivially states that if $D=\emptyset$, then $L$ is an optimal $\{\mathrm{LL}, \mathrm{AP}, \mathrm{P}, \mathrm{LP}\}$-solution, and LL implies $\underline{D}(e, L)=\emptyset$. Proposition 1-2 states that the set of level-0-defaulting firms is always contained in the set of firms defaulting after the CPM is implemented if we impose that this CPM satisfy LL, AP, P and LP.

As a first result, we will question the independence of the axioms in our study in terms of set of defaulting firms. The following proposition shows that Axiom AP is not independent when we consider optimal $\{\mathrm{LP}, \mathrm{LL}, \mathrm{P}, \mathrm{AP}\}$-solutions. ${ }^{6}$

\section{Proposition 2}

Let $0<\alpha, \beta \leq 1$. Let $e=\left(e_{j}\right)_{j \in N} \in \mathcal{E}$ and $L=\left(L_{i, j}\right)_{i, j \in N} \in \mathcal{L} . D \in 2^{N}$ is an optimal $\{L P, L L, P, A P\}$-solution to $(e, L)$ if and only if $D$ is an optimal $\{L P, L L, P\}$-solution to $(e, L)$.

Notice that Proposition 2 deals only with optimal solutions and not with solutions in general. Obviously, the only if part of Proposition 2 still holds if the optimality feature is not considered. However, Example 1 shows that the if part of Proposition 2 does not hold when we consider solutions that are not optimal.

Example 1 Let $0<\alpha, \beta \leq 1$. Let $N=2, e=(2 / \alpha, 2 / \alpha)$ and $L=\left(\begin{array}{ll}0 & 1 \\ 1 & 0\end{array}\right)$. Let us consider the CPM $X=\left(\begin{array}{ll}0 & 1 \\ 0 & 0\end{array}\right) . D(L, X)=\{2\}$, and $X$ satisfies LP, $\mathrm{P}$ and LL. Hence, $\{2\}$ is a $\{\mathrm{LP}, \mathrm{LL}, \mathrm{P}\}$-solution to $(e, L)$. However, it is straightforward to check that $\{2\}$ is not a $\{\mathrm{LP}, \mathrm{LL}, \mathrm{P}, \mathrm{AP}\}$-solution to $(e, L)$. Indeed, if $X^{\prime}=\left(\begin{array}{cc}0 & X_{1,2}^{\prime} \\ X_{1,2}^{\prime} & 0\end{array}\right)$ were to satisfy LP, LL, P and AP and $D\left(L, X^{\prime}\right)=\{2\}$,

\footnotetext{
${ }^{6}$ Notice that we are not claiming that Axiom AP is not independent in some Eisenberg and Noe (2001)'s results dealing with CPMs rather than sets of defaulting firms.
} 
we should have $X_{1,2}^{\prime}=1$ and $2+\beta=X_{2,1}^{\prime}$. In this case, $X^{\prime}$ would not satisfy LP.

Notice however that, in this case, $\emptyset$ is both an optimal $\{L P, L L, P, A P\}$-solution and an optimal $\{\mathrm{LP}, \mathrm{LL}, \mathrm{P}\}$-solution to $(e, L)$.

Proposition 3 shows that when an optimal $\{L P, L L, P, A P\}$-solution is found to a problem with $\alpha=\beta=1$, removing Axiom LP does not help finding smaller (in the sense of inclusion) optimal solutions.

\section{Proposition 3}

Let $\alpha=\beta=1$. Let $e=\left(e_{j}\right)_{j \in N} \in \mathcal{E}$ and $L=\left(L_{i, j}\right)_{i, j \in N} \in \mathcal{L}$. If $D \in 2^{N}$ is an optimal $\{L P, L L, P\}$-solution to $(e, L)$, then $D$ is an optimal $\{L L, P\}$-solution to $(e, L)$.

Let us clarify Proposition 3 and elaborate on it. Let us consider an operating cash flow vector $e$ and a nominal liability matrix $L$ and let $D \in 2^{N}$ be an optimal $\{\mathrm{LP}, \mathrm{LL}, \mathrm{P}\}$ solution to $(e, L)$. By definition, it means that there exists a $\mathrm{CPM} X \in \mathcal{L}$ satisfying $\mathrm{P}$, LP and LL and such that $D(L, X)=D$. Obviously, because $X$ satisfies $\mathrm{P}$ and LL, $D$ is also a $\{\mathrm{LL}, \mathrm{P}\}$-solution to $(e, L)$. However, since we removed axiom LP, and hence some constraints on the CPM, we could hope that there exists another CPM $X^{\prime} \in \mathcal{L}$ satisfying LL and $\mathrm{P}$ only and such that $D\left(L, X^{\prime}\right) \subset D$. This is what Proposition 3 forbids: relaxing Axiom LP does not allow to find a smaller solution to $(e, L)$.

Notice that Proposition 3 does not necessarily hold when $\alpha$ and $\beta$ can be smaller than 1 as can be seen in Example 2

Example 2 Let $\alpha=\beta=1 / 2$. Let $N=4, e=(1,3,8,9)$ and $L=$

$$
\left(\begin{array}{cccc}
0 & 6 & 1 & 0 \\
6 & 0 & 9 & 7 \\
10 & 8 & 0 & 3 \\
0 & 4 & 3 & 0
\end{array}\right)
$$


Let us consider CPM $X=\left(\begin{array}{cccc}0 & 51 / 7 & 17 / 14 & 0 \\ 6 & 0 & 9 & 7 \\ 10 & 8 & 0 & 3 \\ 0 & 4 & 3 & 0\end{array}\right)$. It is straightforward to check that $X$ satisfies $\mathrm{P}, \mathrm{LL}$ (and AP) and is such that $D(L, X)=\{1\}$ and $\emptyset$ is not an $\{\mathrm{LL}, \mathrm{P}\}$-solution to $(e, L)$. Hence, $\{1\}$ is an optimal $\{\mathrm{LL}, \mathrm{P}\}$-solution to $(e, L)$.

Let us consider CPM $X_{1}=\left(\begin{array}{cccc}0 & 6 x_{1} & x_{1} & 0 \\ 6 x_{2} & 0 & 9 x_{2} & 7 x_{2} \\ 10 x_{3} & 8 x_{3} & 0 & 3 \\ 0 & 4 & 3 & 0\end{array}\right)$ with $x_{1}=3353 / 11162$ $x_{2}=1685 / 11162$ and $x_{3}=2567 / 11162$. A bit of calculation shows that $X_{1}$ satisfies LP, P, LL (and AP) and is such that $D\left(L, X_{1}\right)=\{1,2,3\}$.

By Proposition 1-2, $\{1,3\}$ is not a $\{\mathrm{LP}, \mathrm{P}, \mathrm{LL}, \mathrm{AP}\}$-solution to $(e, L)$. Hence, it is not an optimal $\{\mathrm{LP}, \mathrm{P}, \mathrm{LL}, \mathrm{AP}\}$-solution to $(e, L)$. Then, by Proposition 2 , it is not an optimal $\{\mathrm{LP}, \mathrm{P}, \mathrm{LL}\}$-solution to $(e, L)$.

Let us assume that $\{1,2\}$ is a $\{L P, P, L L\}$-solution to $(e, L)$. Then, there exists $X_{2}=\left(\begin{array}{cccc}0 & 6 x_{1} & x_{1} & 0 \\ 6 x_{2} & 0 & 9 x_{2} & 7 x_{2} \\ 10 & 8 & 0 & 3 \\ 0 & 4 & 3 & 0\end{array}\right)$ satisfying LP, P and LL and such that $D\left(L, X_{2}\right)=\{1,2\}$. By $\mathrm{P}$ and definition of $D\left(L, X_{2}\right)$ we must have $x_{1}, x_{2}<1$. Then, we have for firm $3, x_{1}+9 x_{2}+3+8-21<0$. Then, $X_{2}$ does not satisfy LL. Then, $\{1,2\}$ is not a $\{L P, P, L L\}$-solution to $(e, L)$. With the same type of reasoning, we can show that no singleton is a $\{\mathrm{LP}, \mathrm{P}, \mathrm{LL}\}$-solution to $(e, L)$.

Let us assume that $\{2,3\}$ is an optimal $\{\mathrm{LP}, \mathrm{P}, \mathrm{LL}\}$-solution to $(e, L)$. By Proposition $2,\{2,3\}$ is an optimal $\{\mathrm{LP}, \mathrm{P}, \mathrm{LL}, \mathrm{AP}\}$-solution to $(e, L)$ Then, there exists $X_{3}=\left(\begin{array}{cccc}0 & 6 & 1 & 0 \\ 6 x_{2} & 0 & 9 x_{2} & 7 x_{2} \\ 10 x_{3} & 8 x_{3} & 0 & 3 x_{3} \\ 0 & 4 & 3 & 0\end{array}\right)$ satisfying LP, P, LL, AP and such that $D\left(L, X_{3}\right)=\{2,3\}$. Since $X_{3}$ satisfies AP, we must have $\left\{\begin{array}{l}1 / 2\left(3+6+4+8 x_{3}\right)-22 x_{2}=0 \\ 1 / 2\left(8+1+3+9 x_{2}\right)-21 x_{3}=0\end{array}\right.$. After computation, we must have $x_{2}=107 / 296$ and $x_{3}=215 / 592$. Computing 
the value of the equity of $1,6.107 / 296+10.215 / 592+1-7=-118 / 592<0$.

Hence, $X_{3}$ does not satisfy LL. Hence, $\{2,3\}$ is not an optimal $\{L P, P, L L\}-$ solution to $(e, L)$.

Then, $\{1,2,3\}$ is an optimal $\{\mathrm{LP}, \mathrm{P}, \mathrm{LL}\}$-solution to $(e, L)$ and still, $\{1\}$ is an optimal $\{\mathrm{LL}, \mathrm{P}\}$-solution to $(e, L)$.

Notice that as straightforward corollary to Proposition 3, the result can be also stated when optimal $\{\mathrm{LL}, \mathrm{P}, \mathrm{AP}\}$-solution to $(e, L)$ are looked for.

\section{Corollary 1}

Let $\alpha=\beta=1$. Let $e=\left(e_{j}\right)_{j \in N} \in \mathcal{E}$ and $L=\left(L_{i, j}\right)_{i, j \in N} \in \mathcal{L}$. If $D \in 2^{N}$ is an optimal $\{L P, L L, P\}$-solution to $(e, L)$, then, $D$ is an optimal $\{L L, P, A P\}$-solution to $(e, L)$.

Finally, notice also that Proposition 3 does not state that LP is irrelevant when studying optimal $\{\mathrm{LP}, \mathrm{LL}, \mathrm{P}\}$-solutions (or optimal $\{\mathrm{LP}, \mathrm{LL}, \mathrm{P}, \mathrm{AP}\}$-solutions) and when $\alpha=\beta=1$. Indeed, Proposition 4 shows that the converse of Proposition 3 (and of Corollary 1) is not true.

\section{Proposition 4}

Let $\alpha=\beta=1$. Let $e=\left(e_{j}\right)_{j \in N} \in \mathcal{E}$ and $L=\left(L_{i, j}\right)_{i, j \in N} \in \mathcal{L}$.

1) Let $D \in 2^{N}$ be an optimal $\{L L, P\}$-solution to $(e, L)$. D is not necessarily an optimal $\{L P, L L, P\}$-solution to $(e, L)$.

2) Let $D \in 2^{N}$ be an optimal $\{L L, P, A P\}$-solution to $(e, L) . D$ is not necessarily an optimal $\{L P, L L, P\}$-solution to $(e, L)$.

In order to prove Proposition 4, let us consider the following example.

Example 3 Let $N=\{1,2,3,4\}, e=(1,2,2,9)$ and $L=\left(\begin{array}{cccc}0 & 1 & 0 & 0 \\ 2 & 0 & 1 & 2 \\ 1 & 1 & 0 & 1 \\ 1 & 0 & 0 & 0\end{array}\right)$. 
Let us define CPM $X_{1}=\left(\begin{array}{cccc}0 & 5 & 0 & 0 \\ 2 & 0 & 1 & 2 \\ 1 & 1 & 0 & 1 \\ 1 & 0 & 0 & 0\end{array}\right)$.

It is straightforward to check that $X_{1}$ satisfies LL and P. Also, we have, by definition, $D\left(L, X_{1}\right)=\{1\}$.

It is also straightforward to check that there exists no $X \in \mathcal{L}$ such that $D(L, X)=\emptyset$. Then, $\{1\}$ is an optimal $\{\mathrm{LL}, \mathrm{P}\}$-solution to $(e, L)$.

Let us check that there exists no $X=\left(X_{i, j}\right)_{i, j \in N} \in \mathcal{L}$ satisfying LP, P, LL and such that $D(L, X)=\{1\}$. By LL, we should have $X_{N, 2}+e_{2}-X_{2, N} \geq 0$. Then, by $\mathrm{P}, 1+X_{1,2}+2-5 \geq 0$ which cannot be satisfied if $X_{1,2} \leq 1$, imposed by LP.

An optimal $\{\mathrm{LL}, \mathrm{P}, \mathrm{LP}\}$-solution is $\{2,3\}$ with $X_{2}=\left(\begin{array}{cccc}0 & 1 & 0 & 0 \\ 22 / 14 & 0 & 11 / 14 & 22 / 14 \\ 13 / 14 & 13 / 14 & 0 & 13 / 14 \\ 1 & 0 & 0 & 0\end{array}\right)$. It is unique as a corollary of some results of Eisenberg and Noe (2001).

As can be seen from Example 3, imposing that a CPM $X$ satisfy LL and $\mathrm{P}$ with $\alpha=\beta=1$ only may have the consequence that a firm, not initially in $\underline{D}(e, L)$ (firm 1 in this case) be "sacrificed" in order to have firms in $\underline{D}(e, L)$ not defaulting (firm 2 in this case). This may be a normative undesirable consequence of imposing only LL and P. In order to prevent this, we introduce Axiom I.

\section{Axiom 5 (Inclusion of Level-0-Defaulting Firms, I)}

Let $e=\left(e_{j}\right)_{j \in N} \in \mathcal{E}$ and $L=\left(L_{i, j}\right)_{i, j \in N} \in \mathcal{L}$. Let $X \in \mathcal{L}$ be a clearing payment matrix. $X$ satisfies Inclusion of Level-0-Defaulting Firms if

$$
\underline{D}(e, L) \subseteq D(L, X) .
$$

As a corollary to Proposition 3 and Proposition 1-2, we have the following result that states that we cannot improve (in the sense of finding a smaller set with respect to inclusion) an optimal $\{\mathrm{LP}, \mathrm{LL}, \mathrm{P}\}$-solution when searching for an optimal $\{\mathrm{LL}, \mathrm{P}, \mathrm{I}\}$-solution. 


\section{Corollary 2}

Let $\alpha=\beta=1$. Let $e=\left(e_{j}\right)_{j \in N} \in \mathcal{E}$ and $L=\left(L_{i, j}\right)_{i, j \in N} \in \mathcal{L}$. If $D \in 2^{N}$ is an optimal $\{L P, L L, P\}$-solution to $(e, L)$, then, $D$ is an optimal $\{L L, P, I\}$-solution to $(e, L)$.

However, the following proposition shows that it is the only positive result when considering Axiom I.

\section{Proposition 5}

Let $\alpha=\beta=1$. Let $e=\left(e_{j}\right)_{j \in N} \in \mathcal{E}$ and $L=\left(L_{i, j}\right)_{i, j \in N} \in \mathcal{L}$.

1. Let $D \in 2^{N}$ be an optimal $\{L L, P, I\}$-solution to $(e, L)$. $D$ is not necessarily an optimal $\{L P, L L, P\}$-solution to $(e, L)$.

2. Let $D \in 2^{N}$ be an optimal $\{L L, P, I\}$-solution to $(e, L)$. $D$ is not necessarily an optimal $\{L L, P\}$-solution to $(e, L)$.

3. Let $D \in 2^{N}$ be an optimal $\{L L, P\}$-solution to $(e, L)$. $D$ is not necessarily an optimal $\{L L, P, I\}$-solution to $(e, L)$.

\section{Conclusion}

In this paper, we provide an axiomatic analysis of the set of requirements which defines a Clearing Payement Matrix in the framework proposed by Eisenberg and Noe (2001). This set consists of four axioms: Limited Liability (LL), Absolute Priority (AP), Proportionality (P) and Limited Payment (LP). The first three axioms are explicitly stated in Eisenberg and Noe (2001), the last one is implicit. We investigate the consequences of these axioms on the optimal sets of defaulting firms, i.e., the set of firms that are defaulting for a CPM that satisfies the given axioms and that are minimal in the sense of inclusion.

We first show that AP is not independent of the other three axioms when studying the optimal sets of defaulting firms. All the optimal defaulting sets (in the sense of inclusion) for which a CPM satisfies LL, P and LP is an optimal defaulting sets for which a CPM 
satisfies LL, P, LP and AP (this results extends in the direction proposed by Rogers and Veraart (2013)). Then, the optimality feature of these sets is unchanged whether AP is imposed or not. Then, if one's goal is to try to save as many firms as possible - without adding extra money, of course - AP can be removed from the imposed requirements at no cost and no gain.

Second, we show that further removing LP has a more subtle consequence. On the one hand, it does not allow to reduce further the set of defaulting firms compatible with the initial framework of Eisenberg and Noe (2001). On the other hand, there exist optimal defaulting sets that satisfy LL and P but that do not satisfy LL, LP and P. Then, removing LP could possibly lead to find optimal sets of defaulting firms which are different from those provided in the initial framework. This result questions the relevance and justification of LP and its link to some legal requirements.

The two above results are "universal" in the sense that they hold whatever the structure of the relationships between the financial institutions. We provide several further counterexamples which basically show that no other universal results can be derived from other weakening of the set of axioms.

We can see two limitations to our analysis. First, we focused on the set of defaulting firms. Hence, we do not claim, for instance, that the CPM is unchanged when AP is removed. In particular, we do not claim uniqueness of the CPM leading to our results in terms of sets of defaulting firms. This certainly implies a weakness of our results in terms of operational utility. In this line, this study should be seen more as a normative one than a positive one.

Second, our study builds on the axioms introduced in Eisenberg and Noe (2001). These axioms have been debated extensively in the literature and it is left for further research to study how our results are modified when some other - and weaker - axioms are considered. As an example, we kept the strong proportionality requirement (axiom P) along the whole 
paper. It is not clear what results could be derived if we introduce seniority rules (as it is done by Elsinger et al. (2006)), for instance.

Finally, we would like to conclude by saying that our paper shows that weakening the set of constraints given in Eisenberg and Noe (2001) can lead to some new results in terms of sets of defaulting firms. In the present paper, we only compared those sets with the inclusion ranking. However, it may be the case that some of those sets present some appealing features when the rank to compare sets of firms is more complete. This line of study is left for the future. 


\section{References}

Acemoglu, D., Ozdaglar, A., and Tahbaz-Salehi, A. (2015). Systemic risk and stability in financial networks. American Economic Review, 105(2):564-608.

Alessandri, P., Gai, P., Kapadia, S., Mora, N., and Puhr, C. (2009). Towards a framework for quantifying systemic stability. International Journal of Central Banking, 5(3):47-81.

Allen, F. and Gale, D. (2000). Financial contagion. Journal of Political Economy, 108(1):133.

Battiston, S., Delli Gatti, D., Gallegati, M., Greenswald, B. C., and Stiglitz, J. E. (2012a). Default cascades: When does risk diversification increase stability? Journal of Financial Stability, 8(3):138-149.

Battiston, S., Delli Gatti, D., Gallegati, M., Greenswald, B. C., and Stiglitz, J. E. (2012b). Liaisons dangereuses: Increasing connectivity, risk sharing, and systemic risk. Journal of Economic Dynamics and Control, 36(8):1121-1141.

Eisenberg, L. and Noe, T. H. (2001). Systemic risk in financial systems. Management Science, 47(2):236-249.

Elsinger, H., Lehar, A., and Summer, M. (2006). Risk assessment for banking systems. Management Science, 52(9):1301-1314.

Gauthier, C., Lehar, A., and Souissi, M. (2012). Macroprudential capital requirements and systemic risk. Journal of Financial Intermediation, 21(4):594-618.

Georg, C.-P. (2013). The effect of the interbank network structure on contagion and common shocks. Journal of Banking and Finance, 37(7):2216-2228.

Glasserman, P. and Peyton Young, B. (2015). How likely is contagion in financial networks? Journal of Banking and Finance, 50:383-399.

Helmut, E. (2009). Financial networks, cross holdings, and limited liability. Working $\underline{\text { Papers from Oesterreichische Nationalbank (Austrian Central Bank). }}$

Nier, E., Yang, J., Yorulmazer, T., and Alentorn, A. (2007). Network models and financial stability. Journal of Economic Dynamics and Control, 31(6):2033-2060. 
Rogers, L. and Veraart, L. A. (2013). Failure and rescue in an interbank network. Management Science, 59(4):882-898.

Shin, H. S. (2009). Securitisation and financial stability. Economic Journal, 119(536):309 332.

Upper, C. (2011). Simulation methods to assess the danger of contagion in interbank markets. Journal of Financial Stability, 7(3):111-125. 


\section{A Proof of Proposition 1}

Let $0<\alpha, \beta \leq 1$. Let $e=\left(e_{j}\right)_{j \in N} \in \mathcal{E}$ and $L=\left(L_{i, j}\right)_{i, j \in N} \in \mathcal{L}$. The set of $\{\mathrm{LL}, \mathrm{AP}, \mathrm{P}, \mathrm{LP}\}$-solutions to $(e, L)$ being non-empty is a corollary of Rogers and Veraart (2013).

\section{A.1 Proof of Proposition 1-1}

If: Let us assume $\underline{D}(e, L)=\emptyset$. The proof is straightforward by showing that $L$ satisfies LL, AP, P and LP.

Only if: Let us assume that the empty set is a $\{\mathrm{LL}, \mathrm{AP}, \mathrm{P}, \mathrm{LP}\}$-solutions to $(e, L)$, then, there exists $X \in \mathcal{L}$ such that $D(L, X)=\emptyset$. By definition, $X=L$ and hence, $\underline{D}(e, L)=\emptyset$ follows from LL.

\section{A.2 Proof of Proposition 1-2}

Let $X=\left(X_{i, j}\right)_{i, j \in N} \in \mathcal{L}$ be a CPM satisfying LL, AP, P and LP and such that $D(L, X)$ is an optimal $\{\mathrm{LL}, \mathrm{AP}, \mathrm{P}, \mathrm{LP}\}$-solutions to $(e, L)$. Let us assume that we can consider $k \in N$ such that $k \in \underline{D}(e, L)$ and $k \notin D(L, X)$.

Because $X$ satisfies LL, we have

$$
e_{k}+X_{N, k}-X_{k, N} \geq 0
$$

. Because $X$ satisfies $\mathrm{P}$ and $\mathrm{LP}, \forall i \in N, X_{N, k} \leq L_{N, k}$ and by definition of $D(L, X)$, $X_{k, N}=L_{k, N}$ Combining, we must have

$$
e_{k}+L_{N, k}-L_{k, N} \geq 0
$$

. This contradicts the assumption that $k \in \underline{D}(e, L)$. 


\section{B Proof of Proposition 2}

\section{LEMMA 1}

Let $0<\alpha, \beta \leq 1$. Let $e=\left(e_{j}\right)_{j \in N} \in \mathcal{E}$ and $L=\left(L_{i, j}\right)_{i, j \in N} \in \mathcal{L}$. Let $X \in \mathcal{L}$ satisfying LP, $L L, P$ be such that $D(L, X)$ is an optimal $\{L P, L L, P\}$-solution to $(e, L)$. Then, there exists $X^{\prime} \in \mathcal{L}$ satisfying $L P, L L, P, A P$ and such that $D\left(L, X^{\prime}\right)=D(L, X)$.

Proof of Lemma 1: Let $X=\left(X_{i, j}\right)_{i, j \in D}$ satisfy LP, LL, P and such that $D(L, X)$ is an optimal $\{\mathrm{LP}, \mathrm{LL}, \mathrm{P}\}$-solution to $(e, L)$. If $D(L, X)=\emptyset, \mathrm{AP}$ is trivially satisfied and the lemma is proved with $X^{\prime}=X$. Then, let us assume $D(L, X) \neq \emptyset$. In the remainder of the proof, let us write $D=D(L, X)$ and $N D=N D(L, X)$. Because $X$ satisfies LP,

$$
\forall i \in D, X_{i, N} \leq L_{i, N}
$$

Moreover, because $X$ satisfies LL and $\mathrm{P}$,

$$
\begin{gathered}
\forall i \in D, X_{i, N}-\beta . \sum_{j \in D} \frac{L_{j, i}}{L_{j, N}} X_{j, N} \leq \alpha . e_{i}+\beta . \sum_{j \in N D} L_{j, i} \\
\forall i \in N D, L_{i, N}-\sum_{j \in D} \frac{L_{j, i}}{L_{j, N}} X_{j, N} \leq e_{i}+\sum_{j \in N D} L_{j, i}
\end{gathered}
$$

Now, let us consider the solution to the following linear program:

$$
\begin{array}{ll}
T=\arg \max _{\left(X^{\prime}{ }_{i, N}\right)_{i \in D}} \sum_{i \in D} X^{\prime}{ }_{i, N} & \\
\text { subject to: } & \\
0 \leq X^{\prime}{ }_{i, N} \leq L_{i, N} & \forall i \in D \\
X^{\prime}{ }_{i, N}-\beta \cdot \sum_{j \in D} \frac{L_{j, i}}{L_{j, N}} X^{\prime}{ }_{j, N} \leq \alpha \cdot e_{i}+\beta \cdot \sum_{j \in N D} L_{j, i} & \forall i \in D \\
L_{i, N}-\sum_{j \in D} \frac{L_{j, i}}{L_{j, N}} X^{\prime}{ }_{j, N} \leq e_{i}+\sum_{j \in N D} L_{j, i} & \forall i \in N D
\end{array}
$$

Let us define $X^{\mid D}=\left(X_{i, N}^{\mid D}\right)_{i \in D}$ with $\forall i \in D, X_{i, N}^{\mid D}=X_{i, N}$. By Equations 1 and $2, X^{\mid D}$ satisfies the constraints of the program in Equation 3. Moreover, these constraints clearly define a compact set. Hence, the program in Equation 3 has a non empty set of solutions. Let $\bar{X}=\left(\bar{X}_{i, N}\right)_{i \in D}$ be a such a solution. 
Let us define $\overline{X^{N}}=\left({\overline{X^{N}}}_{i, j}\right)_{i, j \in N}$ be defined as $\forall i \in N, i \in D \Rightarrow{\overline{X^{N}}}_{i, j}=\bar{X}_{i, N} \frac{L_{i, j}}{L_{i, N}}$ and $i \in N D \Rightarrow{\overline{X^{N}}}_{i, j}=L_{i, j}$.

It is straightforward to check that $\forall i \in D, \bar{X}_{i, N}=L_{i, N}$ or $\bar{X}_{i, N}-\beta \cdot \sum_{j \in D} \frac{L_{j, i}}{L_{j, N}} \bar{X}_{j, N}=$ $\alpha . e_{i}+\beta . \sum_{j \in N D} L_{j, i}$ (otherwise, increasing $\bar{X}_{i, N}$ by a quantity small enough so that the constraints of Equation 3 are still satisfied is possible and contradicts the assumption that $\bar{X}$ is a solution to Equation 3). If $\exists i \in D, \bar{X}_{i, N}=L_{i, N}$, then $\overline{X^{N}}$ is such that $D\left(L, \overline{X^{N}}\right) \subset D$ and it is straightforward from the constraints of Equation 3 to check that $\overline{X^{N}}$ satisfies LP, $\mathrm{P}$ and $\mathrm{LL}^{7}$. Hence, a contradiction with the fact that $D$ is an optimal $\{\mathrm{LP}, \mathrm{LL}, \mathrm{P}\}$-solution to $(e, L)$. On the contrary, if $\forall i \in D, \bar{X}_{i, N}-\beta . \sum_{j \in D} \frac{L_{j, i}}{L_{j, N}} \bar{X}_{j, N}=\alpha . e_{i}+\beta . \sum_{j \in N D} L_{j, i}$, then $\overline{X^{N}}$ satisfies AP and the lemma is proved with $X^{\prime}=\overline{X^{N}}$.

\section{B.1 Proof of Proposition 2}

Let $0<\alpha, \beta \leq 1$. Let $e=\left(e_{j}\right)_{j \in N} \in \mathcal{E}$ and $L=\left(L_{i, j}\right)_{i, j \in N} \in \mathcal{L}$.

1) Let $D \in 2^{N}$ be an optimal $\{\mathrm{LP}, \mathrm{LL}, \mathrm{P}, \mathrm{AP}\}$-solution to $(e, L)$. Let us show that $D$ is an optimal $\{\mathrm{LP}, \mathrm{LL}, \mathrm{P}\}$-solution to $(e, L)$.

a) Let us show that $D$ is a $\{$ LP,LL,P $\}$-solution to $(e, L)$. By definition, there exists $X \in \mathcal{L}$ such that

- $X$ satisfies LP, LL, P, AP,

- $D=D(L, X)$.

Then, by definition, $D$ is a $\{$ LP,LL,P $\}$-solution to $(e, L)$.

b) Let us show that $D$ is an optimal $\{\mathrm{LP}, \mathrm{LL}, \mathrm{P}\}$-solution to $(e, L)$. In order to have a contradiction, let us have $D^{\prime} \subset D$ be a $\{\mathrm{LP}, \mathrm{LL}, \mathrm{P}\}$-solution to $(e, L)$. Then, there exists

\footnotetext{
${ }^{7}$ Obviously, since $0<\alpha, \beta \leq 1$, if $L_{i, N}-\beta \cdot \sum_{j \in D} \frac{L_{j, i}}{L_{j, N}} \bar{X}_{j, N} \leq \alpha \cdot e_{i}+\beta \cdot \sum_{j \in N D} L_{j, i}$ then, $L_{i, N}-$ $\sum_{j \in D} \frac{L_{j, i}}{L_{j, N}} \bar{X}_{j, N} \leq e_{i}+\sum_{j \in N D} L_{j, i}$
} 
$X \in \mathcal{L}$ such that

- $X$ satisfies LP, LL, P,

- $D^{\prime}=D(L, X)$.

Then, by Lemma 1 , there exists $X^{\prime} \in \mathcal{L}$ such that

- $X^{\prime}$ satisfies LP, LL, P, AP,

- $D^{\prime}=D\left(L, X^{\prime}\right)$.

Then, $D^{\prime}$ is a $\{\mathrm{LP}, \mathrm{LL}, \mathrm{P}, \mathrm{AP}\}$-solution to $(e, L)$, contradicting the assumption that $D$ is an optimal $\{\mathrm{LP}, \mathrm{LL}, \mathrm{P}, \mathrm{AP}\}$-solution to $(e, L)$.

2) Let $D \in 2^{N}$ be an optimal $\{$ LP,LL,P $\}$-solution to $(e, L)$. Let us show that $D$ is an optimal $\{\mathrm{LP}, \mathrm{LL}, \mathrm{P}, \mathrm{AP}\}$-solution to $(e, L)$.

a) Let us show that $D$ is a $\{\mathrm{LP}, \mathrm{LL}, \mathrm{P}, \mathrm{AP}\}$-solution to $(e, L)$. $D$ being an optimal $\{\mathrm{LP}, \mathrm{LL}, \mathrm{P}\}$-solution to $(e, L)$ implies, by definition, that there exists $X \in \mathcal{L}$ such that

- $P$ satisfies LP, LL, P,

- $D=D(L, X)$.

Then, by Lemma 1 , there exists $P^{\prime} \in \mathcal{L}$ satisfying LP, LL, P, AP and such that $D\left(L, X^{\prime}\right)=$ $D(L, X)$. Hence, by definition, $D$ is a $\{\mathrm{LP}, \mathrm{LL}, \mathrm{P}, \mathrm{AP}\}$-solution to $(e, L)$.

b) Let us show that $D$ is an optimal $\{\mathrm{LP}, \mathrm{LL}, \mathrm{P}, \mathrm{AP}\}$-solution to $(e, L)$. On the contrary, let us have $D^{\prime} \subset D$ be a $\{\mathrm{LP}, \mathrm{LL}, \mathrm{P}, \mathrm{AP}\}$-solution to $(e, L)$. By definition, there exists $X \in \mathcal{L}$ such that

- $X$ satisfies LP, LL, P, AP,

- $D^{\prime}=D(L, X)$.

Then, by definition, $D^{\prime}$ is a $\{\mathrm{LP}, \mathrm{LL}, \mathrm{P}\}$-solution to $(e, L)$ contradicting the fact that $D$ is an optimal $\{\mathrm{LP}, \mathrm{LL}, \mathrm{P}\}$-solution to $(e, L)$. 


\section{Proof of Proposition 3}

Let $\alpha=\beta=1$. Let $e=\left(e_{j}\right)_{j \in N} \in \mathcal{E}$ and $L=\left(L_{i, j}\right)_{i, j \in N} \in \mathcal{L}$. Let $D^{1} \in 2^{N}$ be an optimal $\{\mathrm{LP}, \mathrm{LL}, \mathrm{P}\}$-solution to $(e, L)$. By Proposition $2, D^{1}$ is an optimal $\{\mathrm{LP}, \mathrm{LL}, \mathrm{P}, \mathrm{AP}\}$ solution to $(e, L)$. Assume, in contradiction with the proposition, that $D^{2} \subset D^{1}$ is a $\{\mathrm{LL}, \mathrm{P}\}$-solution to $(e, L)$.

If $D^{2}=\emptyset$, then, LP is trivially satisfied and $D^{2}$ is a $\{\mathrm{LP}, \mathrm{LL}, \mathrm{P}\}$-solution to $(e, L)$, contradicting the assumption that $D^{1}$ is an optimal $\{\mathrm{LP}, \mathrm{LL}, \mathrm{P}\}$-solution to $(e, L)$. Then, let us assume that $D^{2} \neq \emptyset$.

Let $X^{1}=\left(X_{i, j}^{1}\right)_{i, j \in N} \in \mathcal{L}$ be such that

- $X^{1}$ satisfies LP, LL, P, AP,

- $D^{1}=D\left(L, X^{1}\right)$.

$X^{1}$ exists since, by assumption, $D^{1}$ is an optimal $\{\mathrm{LP}, \mathrm{LL}, \mathrm{P}, \mathrm{AP}\}$-solution to $(e, L)$.

Let $\mathcal{X}$ be the set of clearing payment matrices $X \in \mathcal{L}$ such that

- $X$ satisfies LL, $\mathrm{P}$,

- $D^{2}=D(L, X)$.

By assumption that $D^{2}$ is a $\{\mathrm{LP}, \mathrm{LL}, \mathrm{P}\}$-solution to $(e, L), \mathcal{X} \neq \emptyset$. For any $X=\left(X_{i, j}\right)_{i, j \in N} \in$ $\mathcal{X}$, let us define $D^{2+}(X)=\left\{i \in D^{2}, X_{i, N}>X_{i, N}^{1}\right\}, D^{2-}(X)=\left\{i \in D^{2}, X_{i, N}<X_{i, N}^{1}\right\}$, $D^{2=}(X)=\left\{i \in D^{2}, X_{i, N}=X_{i, N}^{1}\right\}$. Let us have $X^{2} \in \mathcal{X}$ such that $\forall X \in \mathcal{X}, \neg\left(D^{2-}(X) \subset\right.$ $\left.D^{2-}\left(X^{2}\right)\right)$. By definition, $D^{2+}\left(X^{2}\right) \cup D^{2-}\left(X^{2}\right) \cup D^{2=}\left(X^{2}\right)=D^{2}$. Moreover, if $D^{2+}\left(X^{2}\right)=\emptyset$, $X^{2}$ obviously satisfies LP which, together with the assumption that $X^{2}$ satisfies $\mathrm{P}$ and LL, contradicts the assumption that $D^{1}$ is an optimal $\{\mathrm{LP}, \mathrm{LL}, \mathrm{P}\}$-solution to $(e, L)$. Hence,

$$
D^{2+}\left(X^{2}\right) \neq \emptyset
$$


1) With a proof similar to the one of Lemma 1, we can show that we can consider, with no loss of generality, that $X^{2}$ is such that $\forall i \in D^{2-}\left(X^{2}\right), X_{N, i}^{2}+e_{i}=X_{i, N}^{2}$.

2) Then, summing over $D^{2-}\left(X^{2}\right)$, one obtains

$$
\begin{aligned}
& L_{N \backslash D^{1}, D^{2-}\left(X^{2}\right)}+L_{D^{1} \backslash D^{2}, D^{2-}\left(X^{2}\right)}+ \\
& X_{D^{2=}\left(X^{2}\right), D^{2-}\left(X^{2}\right)}^{2}+X_{D^{2+}\left(X^{2}\right), D^{2-}\left(X^{2}\right)}^{2}+e_{D^{2-}\left(X^{2}\right)}-X_{D^{2-}\left(X^{2}\right), N \backslash D^{2-}\left(X^{2}\right)}^{2}=0 .
\end{aligned}
$$

Moreover, since $X^{1}$ satisfies $\mathrm{AP}$ and $D^{2-}\left(X^{2}\right) \subseteq D^{2} \subset D^{1}$,

$$
\begin{aligned}
& L_{N \backslash D^{1}, D^{2-}\left(X^{2}\right)}+X_{D^{1} \backslash D^{2}, D^{2-}\left(X^{2}\right)}^{1}+ \\
& X_{D^{2}=\left(X^{2}\right), D^{2-}\left(X^{2}\right)}^{1}+X_{D^{2+}\left(X^{2}\right), D^{2-}\left(X^{2}\right)}^{1}+e_{D^{2-}\left(X^{2}\right)}-X_{D^{2-}\left(X^{2}\right), N \backslash D^{2-}\left(X^{2}\right)}^{1}=0 \text {. }
\end{aligned}
$$

Since $X^{1}$ and $X^{2}$ satisfy $\mathrm{P}$, by definition of $D^{2=}\left(X^{2}\right)$ and $D^{2+}\left(X^{2}\right), X_{D^{2=}\left(X^{2}\right), D^{2-}\left(X^{2}\right)}^{2}=$

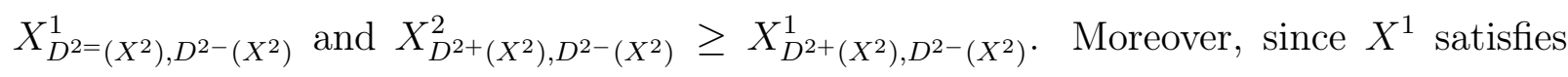
LP, $L_{D_{1} \backslash D_{2}, D^{2-}\left(X^{2}\right)} \geq X_{D_{1} \backslash D_{2}, D^{2-}\left(X^{2}\right)}^{1}$. Then, we necessarily have $X_{D^{2-}\left(X^{2}\right), N \backslash D^{2-}\left(X^{2}\right)}^{2} \geq$ $X_{D^{2-}\left(X^{2}\right), N \backslash D^{2-}\left(X^{2}\right)}^{1}$. Together with the definition of $D^{2-}\left(X^{2}\right)$, we must have $X_{D^{2-}\left(X^{2}\right), N \backslash D^{2-}\left(X^{2}\right)}^{2}=$ $X_{D^{2-}\left(X^{2}\right), N \backslash D^{2-}\left(X^{2}\right)}^{1}=0$. Hence,

$$
\forall i \in D^{2-}\left(P^{2}\right), \forall j \in N \backslash D^{2-}\left(X^{2}\right), X_{i, j}^{1}=X_{i, j}^{2}=0
$$

By Equations 5 and 6, we have

$$
\forall i \in N \backslash D^{2-}\left(X^{2}\right), \forall j \in D^{2-}\left(X^{2}\right), X_{i, j}^{1}=X_{i, j}^{2}=0
$$

3) Since $X^{1}$ satisfies AP,

$$
X_{D^{1} \backslash D^{2}, N \backslash D^{1}}^{1}+X_{D^{2-}\left(X^{2}\right), N \backslash D^{1}}^{1}+X_{D^{2=}\left(X^{2}\right), N \backslash D^{1}}^{1}+X_{D^{2+}\left(X^{2}\right), N \backslash D^{1}}^{1}-L_{N \backslash D^{1}, D^{1}}=e_{N} .
$$

Since $P^{2}$ satisfies LL,

$$
L_{D^{1} \backslash D^{2}, N \backslash D^{1}}+X_{D^{2-}\left(X^{2}\right), N \backslash D^{1}}^{2}+X_{D^{2}=\left(X^{2}\right), N \backslash D^{1}}^{2}+X_{D^{2+}\left(X^{2}\right), N \backslash D^{1}}^{2}-L_{N \backslash D^{1}, D^{1}} \leq e_{N} .
$$

By definition and the fact that $X^{1}$ and $X^{2}$ satisfy $\mathrm{P}, X_{D^{2=}\left(X^{2}\right), N \backslash D^{1}}^{1}=X_{D^{2=}\left(X^{2}\right), N \backslash D^{1}}^{2}$, $X_{D^{2+}\left(X^{2}\right), N \backslash D^{1}}^{2} \geq X_{D^{2+}\left(X^{2}\right), N \backslash D^{1}}^{1}$ and since $X^{1}$ satisfies LP, $L_{D^{1} \backslash D^{2}, N \backslash D^{1}} \geq X_{D^{1} \backslash D^{2}, N \backslash D^{1}}^{1}$. Moreover, by Equation $7, X_{D^{2-}\left(X^{2}\right), N \backslash D^{1}}^{2}=X_{D^{2-}\left(X^{2}\right), N \backslash D^{1}}^{1}=0$. Then,

$$
L_{D^{1} \backslash D^{2}, N \backslash D^{1}}+X_{D^{2}=\left(X^{2}\right), N \backslash D^{1}}^{2}+X_{D^{2+}\left(X^{2}\right), N \backslash D^{1}}^{2}-L_{N \backslash D^{1}, D^{1}}=e_{N},
$$




$$
\begin{gathered}
L_{D^{1} \backslash D^{2}, N \backslash D^{1}}=X_{D^{1} \backslash D^{2}, N \backslash D^{1}}^{1}, \\
X_{D^{2+}\left(X^{2}\right), N \backslash D^{1}}^{2}=X_{D^{2+}\left(X^{2}\right), N \backslash D^{1}}^{1} .
\end{gathered}
$$

Because $X^{1}$ satisfies LP and $\mathrm{P}$ and because $X^{2}$ satisfies $\mathrm{P}$, by definition of $D^{1}$, Equation 10 implies

$$
\forall i \in D^{1} \backslash D^{2}, \forall j \in N \backslash D^{1}, L_{i, j}=X_{i, j}^{2}=X_{i, j}^{1}=0 .
$$

Also, by definition of $D^{2+}\left(X^{2}\right)$, Equation 11 implies

$$
\forall i \in D^{2+}\left(X^{2}\right), \forall j \in N \backslash D^{1}, X_{i, j}^{2}=X_{i, j}^{1}=0 .
$$

Moreover, since $X^{2}$ satisfies LL, Equation 9 implies

$$
\begin{aligned}
& X_{N \backslash D^{2=}\left(X^{2}\right), D^{2=}\left(X^{2}\right)}^{2}+e_{D^{2=}\left(X^{2}\right)}-X_{D^{2=}\left(X^{2}\right), N \backslash D^{2=}\left(X^{2}\right)}^{2}=0, \\
& X_{N \backslash D^{2+}\left(X^{2}\right), D^{2+}\left(X^{2}\right)}^{2}+e_{D^{2+}\left(X^{2}\right)}-X_{D^{2+}\left(X^{2}\right), N \backslash D^{2+}\left(X^{2}\right)}^{2}=0,
\end{aligned}
$$

and

$$
X_{N \backslash\left(D_{1} \backslash D_{2}\right), D_{1} \backslash D_{2}}^{2}+e_{D_{1} \backslash D_{2}}-X_{D_{1} \backslash D_{2}, N \backslash\left(D_{1} \backslash D_{2}\right)}^{2}=0 .
$$

4) We showed above that

$$
X_{N \backslash D^{2=}=\left(X^{2}\right), D^{2=}\left(X^{2}\right)}^{2}+e_{D^{2=}\left(X^{2}\right)}-X_{D^{2=}\left(X^{2}\right), N \backslash D^{2=}\left(X^{2}\right)}^{2}=0,
$$

and by $P^{1}$ satisfying $\mathrm{AP}$, we have

$$
X_{N \backslash D^{2=}\left(X^{2}\right), D^{2=}\left(X^{2}\right)}^{1}+e_{D^{2=}\left(X^{2}\right)}-X_{D^{2=}\left(X^{2}\right), N \backslash D^{2=}\left(X^{2}\right)}^{1}=0 .
$$

The same reasoning as above shows that

$$
\forall i \in D^{1} \backslash D^{2}, \forall j \in D^{2=}\left(X^{2}\right), L_{i, j}=X_{i, j}^{1}=X_{i, j}^{2}=0,
$$

and

$$
\forall i \in D^{2+}\left(X^{2}\right), \forall j \in D^{2=}\left(X^{2}\right), X_{i, j}^{2}=X_{i, j}^{1}=0 .
$$


5) Let us consider $D^{2+}\left(X^{2}\right) \cup\left(D^{1} \backslash D^{2}\right)$. Because $P^{1}$ satisfies AP,

$$
\begin{aligned}
& L_{N \backslash D^{1}, D^{2+}\left(X^{2}\right) \cup\left(D^{1} \backslash D^{2}\right)}+X_{D^{2-}-\left(X^{2}\right), D^{2+}\left(X^{2}\right) \cup\left(D^{1} \backslash D^{2}\right)}^{1}+ \\
& X_{D^{2}=\left(X^{2}\right), D^{2+}\left(X^{2}\right) \cup\left(D^{1} \backslash D^{2}\right)}^{1}-X_{D^{2+}\left(X^{2}\right) \cup\left(D^{1} \backslash D^{2}\right), N \backslash\left(D^{2+}\left(X^{2}\right) \cup\left(D^{1} \backslash D^{2}\right)\right)}^{1}+e_{D^{2+}\left(X^{2}\right) \cup\left(D^{1} \backslash D^{2}\right)}=0 .
\end{aligned}
$$

After simplification using Equations 7, 8, 12, 13, 14, 15

$$
L_{N \backslash D^{1}, D^{2+}\left(X^{2}\right) \cup\left(D^{1} \backslash D^{2}\right)}+X_{D^{2}=\left(X^{2}\right), D^{2+}\left(X^{2}\right) \cup\left(D^{1} \backslash D^{2}\right)}^{1}+e_{D^{2+}\left(X^{2}\right) \cup\left(D^{1} \backslash D^{2}\right)}=0 .
$$

This implies

$$
e_{D^{2+}\left(X^{2}\right) \cup\left(D^{1} \backslash D^{2}\right)}=0 .
$$

and

$$
L_{N \backslash D^{1}, D^{2+}\left(X^{2}\right) \cup\left(D^{1} \backslash D^{2}\right)}+X_{D^{2}=\left(X^{2}\right), D^{2+}\left(X^{2}\right) \cup\left(D^{1} \backslash D^{2}\right)}^{1}=0 .
$$

The same reasoning with $X^{2}$ gives

$$
L_{N \backslash D^{1}, D^{2+}\left(X^{2}\right) \cup\left(D^{1} \backslash D^{2}\right)}+X_{D^{2}=\left(X^{2}\right), D^{2+}\left(X^{2}\right) \cup\left(D^{1} \backslash D^{2}\right)}^{2}=0 .
$$

Equations 17 and 18 imply

$$
\begin{gathered}
\forall i \in N \backslash D^{1}, \forall j \in D^{2+}\left(X^{2}\right) \cup\left(D^{1} \backslash D^{2}\right), L_{i, j}=X_{i, j}^{1}=X_{i, j}^{2}=0, \\
\forall i \in D^{2=}\left(X^{2}\right), \forall j \in D^{2+}\left(X^{2}\right) \cup\left(D^{1} \backslash D^{2}\right), X_{i, j}^{1}=X_{i, j}^{2}=0 .
\end{gathered}
$$

and since $X^{1}$ and $X^{2}$ satisfy LL,

$$
\begin{aligned}
& \forall i \in D^{2+}\left(X^{2}\right) \cup\left(D^{1} \backslash D^{2}\right), \\
& X_{D^{2+}\left(X^{2}\right) \cup\left(D^{1} \backslash D^{2}\right), i}^{1}-X_{i, D^{2+}\left(X^{2}\right) \cup\left(D^{1} \backslash D^{2}\right)}^{1}= \\
& X_{D^{2+}\left(X^{2}\right) \cup\left(D^{1} \backslash D^{2}\right), i}^{2}-X_{i, D^{2+}\left(X^{2}\right) \cup\left(D^{1} \backslash D^{2}\right)}^{2}=0
\end{aligned}
$$

6) By Equation $4, D^{2+}\left(X^{2}\right) \neq \emptyset$ and by definition of $D^{2+}\left(X^{2}\right), \forall k \in D^{2+}\left(X^{2}\right), X_{k, N}^{2}>$ $X_{k, N}^{1} \geq 0$. Moreover, since $X^{2}$ satisfies $\mathrm{P}, \forall k \in D^{2+}\left(X^{2}\right), L_{k, N}>0$. Then, we can consider $k_{\max } \in D^{2+}\left(X^{2}\right)$ such that $\forall k \in D^{2+}\left(X^{2}\right), \frac{X_{k_{\max }, N}^{2}}{L_{k_{\max }, N}} \geq \frac{X_{k, N}^{2}}{L_{k, N}}$.

Assume that $\frac{X_{k_{\max }, N}^{2}}{L_{k_{\max }, N}} \leq 1$. This implies that $X^{2}$ satisfies LP, contradicting the fact that $D^{1}$ is an optimal $\{\mathrm{LP}, \mathrm{P}, \mathrm{LL}\}$-solution. Then, let us consider $\frac{X_{k_{\max }, N}^{2}}{L_{k_{\max }, N}}>1$. 
Now, let us define $X^{\prime}=\left(X^{\prime}{ }_{i, j}\right)_{i, j \in N} \in \mathcal{L}$ as:

$$
\forall i, j \in N, X_{i, j}^{\prime}= \begin{cases}X_{i, j}^{2} \cdot \frac{L_{k_{\max }, N}}{X_{k_{\max }, N}} & , \text { if } i \in D^{2+}\left(X^{2}\right) \cup\left(D^{1} \backslash D^{2}\right), \\ X_{i, j}^{2} & \text { otherwise. }\end{cases}
$$

It is straightforward to check that $X^{\prime}$ satisfies $\mathrm{P}$.

Let us show that $X^{\prime}$ satisfies LL. a) Let $i \in N \backslash\left(D^{2+}\left(X^{2}\right) \cup\left(D^{1} \backslash D^{2}\right)\right)$. Let us define $\pi(i)=X^{\prime}{ }_{N, i}+e_{i}-X_{i, N}^{\prime}$. By definition of $X^{\prime}, \pi(i)=X_{N, i}^{\prime}+e_{i}-X_{i, N}^{2}$. By Equations, 8, 12, 13, 14 and $15, \pi(i)=X^{2}{ }_{N, i}+e_{i}-X^{2}{ }_{i, N}$. Then, LL is satisfied by $i$ for $X^{\prime}$ as it is for $X^{2}$ by assumption. b) Let $i \in D^{2+}\left(X^{2}\right) \cup\left(D^{1} \backslash D^{2}\right)$. Let us define $\pi(i)=X_{N, i}^{\prime}+e_{i}-X_{i, N}^{\prime}$. After simplification using Equations 7, 8, 12, 13, 14, 15, 16,

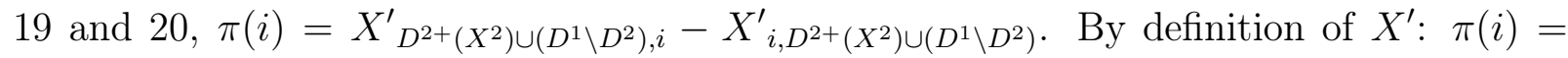
$\frac{L_{k_{\max }, N}}{X_{k_{\max }, N}^{2}} X^{2}{ }_{N, i}-X^{2}{ }_{i, N}$. Then, LL is satisfied by $i$ for $X^{\prime}$ as it is for $X^{2}$ by assumption.

Let us show that $X^{\prime}$ satisfies LP. a) The proof is straightforward for all $i \in N \backslash$ $\left(D^{2+}\left(X^{2}\right) \cup\left(D^{1} \backslash D^{2}\right)\right)$. b) Let $i \in D^{2+}\left(X^{2}\right) . X^{\prime}{ }_{i, N}=X_{i, N}^{2} \cdot \frac{L_{k_{\max }, N}}{X_{k_{\max }, N}^{2}}$. Then, by definition of $k_{\text {max }}, X_{i, N}^{\prime} \leq X_{i, N}^{2} \cdot \frac{L_{i, N}}{X_{i, N}^{2}}=L_{i, N}$. Then, LP is satisfied by $i$. c) Let $i \in D^{1} \backslash D^{2}$. $X_{i, N}^{\prime}=X_{i, N}^{2} \cdot \frac{L_{k_{\max }, N}}{X_{k_{\max }, N}^{2}}=L_{i, N} \cdot \frac{L_{k_{\max }, N}}{X_{k_{\max }, N}^{2}}$. Since, $\frac{L_{k_{\max }, N}}{X_{k_{\max }, N}^{2}}<1, X_{i, N}^{\prime}<L_{i, N}$. Then, LP is satisfied by $i$.

It is straightforward to check that, since $\left(N \backslash D^{1}\right) \cap\left(D^{2+}\left(P^{2}\right) \cup\left(D^{1} \backslash D^{2}\right)\right)=\emptyset$, $D\left(L, X^{\prime}\right) \subseteq D\left(L, X^{1}\right)$. It is also straightforward to check that $k_{\text {max }} \notin D\left(L, X^{\prime}\right)$, whereas by definition, $k_{\max } \in D^{2+}\left(X^{2}\right) \subseteq D\left(L, X^{1}\right)$. Hence, $D\left(L, X^{\prime}\right) \subset D\left(L, X^{1}\right)$ which contradicts the fact that $D^{1}$ is an optimal $\{\mathrm{LP}, \mathrm{P}, \mathrm{LL}\}$-solution. This completes the proof.

\section{Proof of Proposition 5}

Let $N=\{1,2,3,4\}, e=(1,2,3,4)$ and $L=\left(\begin{array}{cccc}0 & 1 & 2 & 1 \\ 1 & 0 & 1 & 2 \\ 1 & 1 & 0 & 1 \\ 0 & 0 & 2 & 0\end{array}\right)$.

We have $\underline{D}(e, L)=\{1\}$. 
Let us define the CPM $X_{1}=\left(\begin{array}{cccc}0 & 6 / 5 & 12 / 5 & 6 / 5 \\ 1 & 0 & 1 & 2 \\ 14 / 5 & 14 / 5 & 0 & 14 / 5 \\ 0 & 0 & 2 & 0\end{array}\right) . X_{1}$ satisfies LL, P and I and $D\left(L, X_{1}\right)=\{1,3\}$ is an optimal $\{\mathrm{LL}, \mathrm{P}, \mathrm{I}\}$-solution to $(e, L)$.

Let us define the CPM $X_{2}=\left(\begin{array}{cccc}0 & 1 & 2 & 1 \\ 1 & 0 & 1 & 2 \\ 8 / 3 & 8 / 3 & 0 & 8 / 3 \\ 0 & 0 & 2 & 0\end{array}\right) . \quad X_{2}$ satisfies LL and $\mathrm{P}$ and $D\left(L, X_{2}\right)=\{3\}$ is an optimal $\{\mathrm{LL}, \mathrm{P}\}$-solution to $(e, L)$.

Let us define the CPM $X_{3}=\left(\begin{array}{cccc}0 & 11 / 15 & 22 / 15 & 11 / 15 \\ 14 / 15 & 0 & 14 / 15 & 28 / 15 \\ 1 & 1 & 0 & 1 \\ 0 & 0 & 2 & 0\end{array}\right) . X_{3}$ satisfies LL, P and LP and $D\left(L, X_{3}\right)=\{1,2\}$ is an optimal $\{\mathrm{LL}, \mathrm{P}, \mathrm{LP}\}$-solution to $(e, L)$.

Then, $D\left(L, X_{1}\right)$ is an optimal $\{\mathrm{LL}, \mathrm{P}, \mathrm{I}\}$-solution to $(e, L)$ but is not an optimal $\{\mathrm{LL}, \mathrm{P}, \mathrm{LP}\}$ solution to $(e, L)$ proving Proposition 5-1. $D\left(L, X_{1}\right)$ is an optimal $\{\mathrm{LL}, \mathrm{P}, \mathrm{I}\}$-solution to $(e, L)$ but is not an optimal $\{\mathrm{LL}, \mathrm{P}\}$-solution to $(e, L)$ proving Proposition 5-2. $D\left(L, X_{2}\right)$ is an optimal $\{\mathrm{LL}, \mathrm{P}\}$-solution to $(e, L)$ but is not an optimal $\{\mathrm{LL}, \mathrm{P}, \mathrm{I}\}$-solution to $(e, L)$ proving Proposition 5-3. 